\title{
Healthy Aging by Staying Selectively Connected: A Mini-Review
}

\author{
Daria Antonenko Agnes Flöel \\ Department of Neurology, NeuroCure Cluster of Excellence, and Center for Stroke Research Berlin, \\ Charité-Universitätsmedizin Berlin, Berlin, Germany
}

\section{Key Words}

Functional connectivity - Functional magnetic resonance imaging · Resting state - Structural connectivity - Diffusion tensor imaging · Fractional anisotropy · Brain networks

\begin{abstract}
Cognitive neuroscience of the healthy aging human brain has thus far addressed age-related changes of local functional and structural properties of gray and white matter and their association with declining or preserved cognitive functions. In addition to these localized changes, recent neuroimaging research has attributed an important role to neural networks with a stronger focus on interacting rather than isolated brain regions. The analysis of functional connectivity encompasses task-dependent and -independent synchronous activity in the brain, and thus reflects the organization of the brain in distinct performance-relevant networks. Structural connectivity in white matter pathways, representing the integrity of anatomical connections, underlies the communication between the nodes of these functional networks. Both functional and structural connectivity within these networks have been demonstrated to change with aging, and to have different predictive values for cognitive abilities in older compared to young adults. Structural degeneration has been found in the entire cerebral white matter
\end{abstract}

with greatest deterioration in frontal areas, affecting whole brain structural network efficiency. With regard to functional connectivity, both higher and lower functional coupling has been observed in the aging compared to the young brain. Here, high connectivity within the nodes of specific functional networks on the one hand, and low connectivity to regions outside this network on the other hand, were associated with preserved cognitive functions in aging in most cases. For example, in the language domain, connections between left-hemisphere language-related prefrontal, posterior temporal and parietal areas were described as beneficial, whereas connections between the left and right hemisphere were detrimental for language task performance. Of note, interactions between structural and functional network properties may change in the course of aging and differentially impact behavioral performance in older versus young adults. Finally, studies using noninvasive brain stimulation techniques like transcranial direct current stimulation (tDCS) to simultaneously modulate behavior and functional connectivity support the importance of 'selective connectivity' of aging brain networks for preserved cognitive functions. These studies demonstrate that enhancing task performance by tDCS is paralleled by increased connectivity within functional networks. In this review, we outline the network perspective on healthy brain aging and discuss recent developments in this field.

\section{KARGER}

E-Mail karger@karger.com

www.karger.com/ger
(C) 2013 S. Karger AG, Basel

0304-324X/14/0601-0003\$39.50/0
Agnes Flöel

Department of Neurology, NeuroCure Cluster of Excellence, and

Center for Stroke Research Berlin, Charité-Universitätsmedizin Berlin

Charitéplatz 1, DE-10117 Berlin (Germany)

E-Mail agnes.floeel@charite.de 


\section{Localized Structural and Functional Changes in Aging}

Cognitive neuroscience of the healthy aging human brain has extensively investigated the association between age-related changes in functional and structural properties of local gray and white matter and declining or preserved cognitive functions. These findings have been comprehensively discussed in previous reviews [e.g., 1, $2]$. Briefly, age-associated shrinkage in grey matter volume and decrease in white matter integrity, especially in prefrontal and medial temporal areas, have been linked to cognitive deficits in various domains [for details, see 1]. The degree of age-related structural alterations correlated with the magnitude of behavioral deficits in some but not all studies. With regard to age-related alterations in localized functional activations, increased bilateral, mostly prefrontal, activation in older compared to young adults has been consistently reported [for a review, see 1]. However, its exact interpretation remains to be established. While some authors have linked these changes to better performance and thus postulated compensatory mechanisms for increased activation, others have demonstrated an association between reduced performance and increased activation, and therefore suggested inefficient or even detrimental over-recruitment of brain areas with regard to function. Moreover, decreased task-related activations have also been observed in aging, for example in the mediotemporal and sensory regions, in most but not all cases associated with impaired performance [for details, see 1]. In sum, the predictive value of both increased and decreased functional activation for successful cognitive aging is still under debate $[1,2]$.

Previous inconsistent findings may at least in part be due to the complex interplay and interdependency of deterioration in different brain areas, with behavioral decline being dependent on the specific combination of structural and functional alterations of various regions [3]. For instance, age-related decreased activity in posterior occipital areas may be compensated for by increased activity in prefrontal brain areas instantiated by some older adults, as shown by Davis et al. [3]. These studies suggest that the examination of isolated regional changes might not suffice to explain the neural mechanisms of age-related cognitive decline. Instead, they support the necessity of investigating interacting brain areas to elucidate the neural mechanisms underlying altered cognitive functions associated with aging. Note that in the context of the present review, 'brain networks' are defined as 'topological organization of coordinated systems compris- ing specific brain areas with structured connectivity patterns which function together to promote particular cognitive functions' [e.g., 4-6]. The two organizing principles of these networks are information segregation and integration [e.g., 6, 7]. Examples are networks supporting sensory, motor, executive, memory or language functions.

\section{Structural and Functional Brain Networks in the Context of Age-Related Cognitive Decline}

Recent neuroimaging studies have drawn increased attention to the importance of neural communication within brain networks for cognitive performance $[2,8]$. They emphasize the importance of changes in network activity, in addition to altered local degeneration and activation, in the context of age-related cognitive decline [e.g., 4].

Investigating the relationship between functional brain networks, the underlying microstructure in connecting white matter pathways, and cognitive function is particularly interesting in understanding the decline or preservation of cognitive functions in the healthy older human brain [for a recent review, see 2]. In addition, there is converging evidence that neurodegenerative diseases such as Alzheimer's disease also show network alterations as a hallmark of disease progression [e.g., 9]. In addition, cognitive decline in the form of mild cognitive impairment is associated with connectivity changes reflecting atypical network functioning [10].

\section{Structural Brain Networks}

Structural connectivity can be measured with diffusion tensor imaging and assessed by quantifying microstructural properties of white matter (e.g., fractional anisotropy or mean diffusivity) or by reconstructing and characterizing white matter pathways $[8,11]$. These pathways form the anatomical basis for efficient information transfer in the brain. Age-related disruptions will be evident in damaged microstructure and deteriorated tracts, which are smaller in their extent or volume. White matter fiber pathways have been found to deteriorate in aging throughout the entire brain, with the largest alteration seen in frontal brain areas $[1,8]$. Deteriorated white matter pathways may then be less able to effectively transfer information, thus affecting the efficiency of communication within and between networks they are part of, and ultimately lead to lower cognitive function [6].

For example, Wen et al. [6] found that brain global structural network efficiency, as assessed by graph theo- 
retical analysis of white matter integrity, was reduced in aging and predicted a decline in processing speed, visuospatial and executive functions. In addition, local efficiency within specific task-relevant regions had an impact on various functions in older adults. The authors concluded that for efficient functioning, the aging brain has to effectively couple the respective cortical regions into interacting structural networks.

\section{Functional Brain Networks}

Functional connectivity between brain areas reflects the extent to which their activities are synchronized or temporally correlated [12]. It represents the quality of information transfer and functional communication between regions. These temporal correlations can be explained by direct or indirect interactions, i.e., the connection can be provided by either direct white matter pathways or by complex connections between multiple brain areas [12]. Both lower and higher functional connectivity patterns in older versus young individuals have been associated with decreased cognitive functions.

There are various techniques to measure functional connectivity in aging brain networks, including electroencephalography, magnetoencephalography $[10,13]$ and functional magnetic resonance imaging (fMRI) [14] as well as multiple analysis methods, e.g., graph-theoretical [5], non-linear [10] and correlational approaches [12]. Here, we focus on inter-regional correlations in task-related or resting-state, see Fox and Raichle [12] and Ferreira and Busatto [14] for reviews, respectively. Functional connectivity analyses based on task-related fMRI may be confounded by task compliance, performance and difficulty effects, while resting-state fMRI assessments reflect intrinsic network architecture without those confounds [14]. Below, we describe recent studies investigating age-related network alterations and their behavioral relevance. We use the term 'efficiency' to describe intra-network coupling, referring to the successful transmission of information between brain areas belonging to the same functional network. The term 'specificity' is used to describe inter-network coupling, referring to the selectivity of brain networks, i.e., their functional distinction and successful differentiation from other networks.

\section{Beneficial and Detrimental Functional Connections:}

Reduced Efficiency and Specificity in Aging Brain

Networks

Previous studies have shown that in older adults, alterations of the network connectivity patterns that had previously been demonstrated in young adults were associated with cognitive decline $[4,6,7]$. Andrews-Hanna et al. [4] demonstrated that healthy older compared to young adults showed reduced functional connectivity within the default network that was associated with deteriorated white matter as well as poorer behavioral performance on various cognitive domains such as processing speed, memory, and executive functions. They concluded that the age-related disruption in the coordination of large-scale brain systems leads to cognitive decline. Similarly, a recent study by Geerligs et al. [7] investigated communication within the default network as well as within other task-relevant brain networks such as the frontoparietal control and the dorsal attention network, in older and young adults. They found that decreased connectivity within these networks in older compared to young adults was associated with poorer performance on various cognitive domains, including executive functions, working memory and verbal learning. Interestingly, in addition to showing decreased intra-network connectivity, older adults also exhibited increased inter-network connectivity compared to young adults. While in their study the intra-network connectivity decrease was directly correlated with performance, the inter-network connectivity only showed an overall increase in the older compared to the young group, and was interpreted as a general reduction of specificity of functional brain networks. This result is consistent with theories of the aging brain that suggest a functional dedifferentiation resulting from a neural reorganization of excitatory and inhibitory processes in the aging brain [1]. Dedifferentiation refers to the decreased functional selectivity of brain areas in aging, resulting in more diffuse and less specialized patterns of functional connections $[1,15]$. In the study by Geerligs et al. [7], the reason for the lack of a direct correlation between increased inter-network connectivity and decreased performance was unclear. However, such a direct relationship has been demonstrated in young subjects, in whom an inverse correlation between functional connectivity of medial frontal to dorsolateral prefrontal areas and working memory performance was observed [e.g., $16]$.

Given the substantial deterioration of inhibitory processes in the aging brain, which impairs physiological inhibitory interactions particularly between hemispheres [17], it is likely that age-related increases in inter-hemispheric connectivity negatively impact cognition. This has been particularly suggested for highly lateralized functions such as motor or language task performance. Here, lateralized intra-hemispheric network connectivity 
may support superior behavior, while increased interhemispheric functional connectivity (even within functional networks like the motor network) may have a negative impact on function $[17,18]$. For example, Langan et al. [18] demonstrated degenerated anterior callosal fibers - which carry primarily inhibitory functions between the hemispheres - in older compared to young adults. This degeneration was associated with prolonged reaction times in a motor task as well as increased functional connectivity between left and right motor areas [18]. This observation might be explained by a shift from a strong inhibitory communication between the hemispheres in the young brain that restricts functional communication towards lower inter-hemispheric inhibitory tone in the older brain [for a discussion, see 17].

In the language domain, stronger inter-hemispheric functional coupling between prefrontal areas was detrimental for performance in a grammar-learning task in healthy older adults [19]. Here, bilateral white matter integrity in the left and right Brodmann area 44/45 (Broca's area) was positively related to learning performance in older adults. In contrast, higher inter-hemispheric functional coupling of these prefrontal areas predicted poorer performance in older adults. In a subsequent study, the authors confirmed the proposed detrimental influence of inter-hemispheric functional connectivity in older adults in direct comparison to a group of young adults [20]. Functional connectivity within brain networks and performance on a syntactic comprehension task were compared between older and young adults, revealing more intra-network coupling in young compared to older adults that was associated with superior language task performance. In contrast, older adults' language networks showed higher connections to areas outside the core language network implicated in working or semantic memory. This pattern of brain-behavior relationships differed between the age groups, showing a detrimental influence of enhanced inter-hemispheric functional coupling only in older adults.

Recent studies have consistently showed an age-related increase in the recruitment of more general instead of specific functional networks $[21,22]$, associated with lower function of the specific cognitive domain under study. These findings of detrimental effects of increased internetwork connectivity - which are consistent with the idea of neural dedifferentiation in the aging brain $[1,7]$ - are complemented by recent studies showing a negative association between the connection to areas that are not part of the same network and behavioral performance. Our conclusions from recent data complement and refine current theories of age-related cognitive decline [see 1, 7, 17]. Recruitment of additional brain areas (not belonging to the same functional network) may be rather detrimental (in line with 'dedifferentiation theories'). In addition, our conclusions also support certain aspects of 'compensation theories' [1], in the sense that stronger connections between particular nodes of a given functional network in older individuals would yield a beneficial impact on function [20].

In sum, there is evidence for a beneficial impact of higher intra-network connection on behavior - reflecting efficiency of communication within a given brain network, at least within one hemisphere - and lower internetwork connection - reflecting specificity and selectivity of the network under study - for preserved cognitive functions in older adults. Using the example of the language network, these age-related functional network alterations are schematically illustrated in figure 1.

\section{Interacting Brain Networks}

Advanced aging of the human brain is accompanied not only by altered connectivity within networks due to structural damage, but also by changing interrelations of functional and structural network properties $[8,23]$. Particularly, the extent to which lower integrity of white matter microstructure influences functional communication within brain networks might differ between healthy young and older adults, probably becoming even more relevant in the context of age-related cognitive decline. More precisely, white matter integrity would predict functional connectivity in older but not young adults [4]. Especially connections that are deteriorated in older compared to young adults may show correlations to behavioral performance [7]. For example, Chen et al. [24] demonstrated an association between white matter integrity in the genu of the corpus callosum (promoting interhemispheric communication) and functional connectivity between inferior frontal gyri only in older adults.

In case of an intact white matter network and absence of grey matter damage as found in the young brain, information may be effectively transferred between the nodes of networks via the most efficient routes. As the white matter infrastructure, which determines the quality of information transfer, declines in the course of aging, the extent to which brain regions are able to communicate is altered and will ultimately impact cognitive performance [see 8]. Structural damage may impact some 'routes' more than others, leading to inhomogeneity in inter-regional functional communication, with particular 'routes' becoming more relevant for transmitting specific informa- 


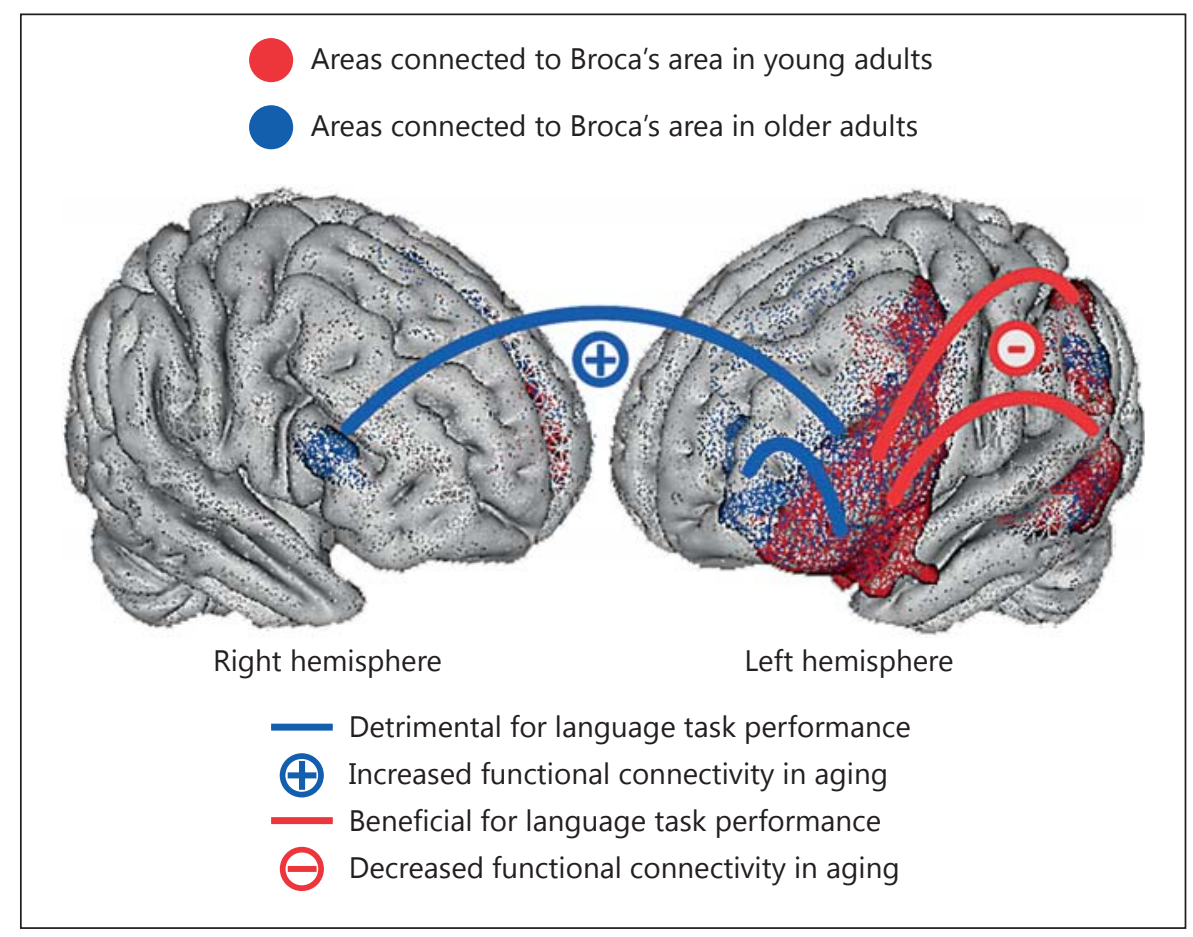

Fig. 1. Reduced network specificity and efficiency in aging. Schematic illustration of age-related alterations of functional coupling within the 'language' network [27] as an example of reduced network specificity and efficiency in aging. Functional networks were derived from seed-based analysis using Brodmann area 44/45 (Broca's area) as the seed of resting-state fMRI data from Antonenko et al. [20]. Brain areas functionally connected to Broca's area are shown in red for young adults and in blue for older adults. There is a substantial overlap of the language network between young and older adults, with the intra-network (mostly left-hemisphere) being stronger in young adults and beneficial for language abilities.
These intra-network connections may decrease in aging due to deterioration of mediating white matter pathways such as the superior longitudinal fasciculus. Inter-network functional connectivity (with right-hemisphere homologues or left-hemisphere areas not included in the 'young' network) may increase due to deteriorated inhibitory white matter pathways such as the anterior corpus callosum, and show a behavioral relevance in older adults. The maintenance of network efficiency (i.e., intra-network coupling) and at the same time specificity (i.e., inter-network coupling) in aging is defined as the ability to 'stay selectively connected' and is assumed to be essential for successful brain aging. tion $[24,25]$. This phenomenon may also increase intraindividual variability in cognitive functions, in addition to an increase in inter-individual variability due to differential white matter deterioration between older adults. Particularly, brain-behavior correlations may change due to age-related complex network reorganizations involving changes in both inhibitory and excitatory processes $[17,20,25,26]$.

For example, as described above for the language domain, the comparison of functional and structural networks revealed different patterns in older and young adults, not only in network properties but also in their association with language task performance [20]. In terms of functional coupling, the strength of intra-network connectivity within the left-hemisphere language network showed a behavioral relevance for syntactic abilities only in young adults. 'Young' networks were characterized by higher connectivity between left-hemisphere prefrontal and inferior parietal and temporal areas, which predicted superior performance. On the contrary, in older adults the inter-network connectivity was more relevant. 'Older' networks were characterized by a substantial increase of connectivity to areas outside the 'young' network, such as left middle frontal, and left and right angular gyri. In addition, these distinctive connections as well as interhemispheric coupling predicted performance in older adults. With regard to structural connectivity, different white matter pathways associated with language abilities were related to each groups' syntactic performance, indicating differential relationships between functional and 
structural connectivity and between connectivity and behavior. Here, young adults' dorsal white matter language pathways [27] predicted syntactic ability and the degree of functional coupling between the connected prefrontal and inferior parietal areas. On the other hand, older adults' ventral white matter language pathways [27] predicted syntactic ability and the degree of functional coupling between connected prefrontal and middle temporal areas [20].

Thereby, age-related differences in brain-behavior relationships might be observed even without significant age-related decline in the respective neural parameters $[20,26]$. This observation supports the assumption that neural reorganization of inhibitory and excitatory processes may be responsible for distinct age-related associations rather than structural decline per se.

In sum, previous studies have focused on age-related connectivity reductions within particular brain networks without investigating potentially interfering connectivity to other networks [see 7], or these studies have only looked at either functional or structural connectivity. In order to adequately account for the complex network reorganizations in aging with their behavioral relevance and inter-relations of structural and functional properties [1], research studies need to integrate functional and structural connectivity as well as a detailed analysis of behavioral performance [8].

\section{Improving Functional Brain Networks}

The classical approach of connectivity studies in the living human brain described above is to assess brain networks in the aging brain with fMRI or diffusion tensor imaging, quantify connectivity within or between network nodes and relate it to behavioral performance. Beyond this correlational approach, the relationship between interacting brain areas and behavioral performance can be probed using interventional approaches such as noninvasive brain stimulation techniques. Examples of such techniques are transcranial magnetic stimulation and transcranial direct current stimulation (tDCS), which may effectively enhance the efficiency of brain networks promoting a particular function [13,28]. For example, in a recent study by Meinzer et al. [29], task-related activity of a semantic fluency paradigm and resting-state functional connectivity were assessed during anodal tDCS over the ventral part of the left inferior frontal cortex, a core area for semantic fluency. Improved behavioral performance was associated with selectively reduced task- related activation in the left ventral inferior frontal gyrus and increased connectivity of the left inferior frontal gyrus and additional major hubs overlapping with the language network [29]. In a subsequent study, the authors applied the same approach to healthy older adults, and showed improved behavioral performance as well as increased network efficiency and specificity in older adults [30]. Importantly, both functional connectivity patterns as well as performance levels approached those found in the control group of young subjects. These results open exciting new avenues to improve brain network efficiency in the aging brain. Future studies should now assess whether repeated anodal tDCS application will lead to sustained modulations of network efficiency and behavior in older individuals, not only with regard to language processing but also targeting other cognitive domains including memory, attention and executive functions.

\section{Conclusions}

Recent studies point towards the necessity for brain regions to stay selectively connected within specific networks - i.e., positively to regions supporting similar functions and negatively to those not strongly involved in the specific task - for maintaining high cognitive function in healthy aging. Consequently, interventions aiming to modulate cognitive functions in aging should be aimed towards enhancing intra-network connectivity, at least within the hemisphere dominant for the task under study, while simultaneously reducing inter-network connectivity. Here, $\mathrm{tDCS}$ provides a promising noninvasive device, a hypothesis to be further explored in studies using repeated stimulation protocols and long-term follow-up.

\section{Acknowledgements}

We thank Dr. Frank Thurm for helpful comments. The research on age-related language network changes and modulation with tDCS described in this review was supported by grants from the Deutsche Forschungsgemeinschaft (Fl 379-8/1, Fl 379-10/1, Fl 379-11/1; and DFG-Exc 257), the Else-Kröner Fresenius Stiftung (2009-141; 2011-119), and the Bundesministerium für Bildung und Forschung (FKZ 0315673A, 01EO0801 and 01GY1144). 


\section{References}

1 Park DC, Reuter-Lorenz P: The adaptive brain: aging and neurocognitive scaffolding. Annu Rev Psychol 2009;60:173-196.

$>2$ Grady C: The cognitive neuroscience of ageing. Nat Rev Neurosci 2012;13:491-505.

-3 Davis SW, Dennis NA, Daselaar SM, Fleck MS, Cabeza R: Que pasa? The posterior-anterior shift in aging. Cereb Cortex 2008;18: 1201-1209.

4 Andrews-Hanna JR, Snyder AZ, Vincent JL, Lustig C, Head D, Raichle ME, Buckner RL: Disruption of large-scale brain systems in advanced aging. Neuron 2007;56:924-935.

$\checkmark 5$ Meunier D, Achard S, Morcom A, Bullmore E: Age-related changes in modular organization of human brain functional networks. Neuroimage 2009;44:715-723.

-6 Wen W, Zhu W, He Y, Kochan NA, Reppermund S, Slavin MJ, Brodaty H, Crawford J, Xia A, Sachdev P: Discrete neuroanatomical networks are associated with specific cognitive abilities in old age. J Neurosci 2011;31: 1204-1212.

7 Geerligs L, Maurits NM, Renken RJ, Lorist MM: Reduced specificity of functional connectivity in the aging brain during task performance. Hum Brain Mapp 2012, E-pub ahead of print.

$>8$ Salat DH: The declining infrastructure of the aging brain. Brain Connect 2011;1:279-293.

$>9$ Greicius MD, Kimmel DL: Neuroimaging insights into network-based neurodegeneration. Curr Opin Neurol 2012;25:727-734.

10 Buldu JM, Bajo R, Maestu F, Castellanos N, Leyva I, Gil P, Sendina-Nadal I, Almendral JA, Nevado A, del-Pozo F, Boccaletti S: Reorganization of functional networks in mild cognitive impairment. PLoS One 2011; 6:e19584.

11 Johansen-Berg $\mathrm{H}$, Behrens TEJ: Diffusion MRI: From Quantitative Measurement to invivo Neuroanatomy. London, Elsevier, 2009.
12 Fox MD, Raichle ME: Spontaneous fluctuations in brain activity observed with functional magnetic resonance imaging. Nat Rev Neurosci 2007;8:700-711.

13 Freitas C, Farzan F, Pascual-Leone A: Assessing brain plasticity across the lifespan with transcranial magnetic stimulation: why, how, and what is the ultimate goal? Front Neurosci 2013;7:42.

14 Ferreira LK, Busatto GF: Resting-state functional connectivity in normal brain aging. Neurosci Biobehav Rev 2013;37:384-400.

15 Baltes PB, Lindenberger U: Emergence of a powerful connection between sensory and cognitive functions across the adult life span: a new window to the study of cognitive aging? Psychol Aging 1997;12:12-21.

16 Hampson M, Driesen N, Roth JK, Gore JC, Constable RT: Functional connectivity between task-positive and task-negative brain areas and its relation to working memory performance. Magn Reson Imaging 2010;28: 1051-1057.

17 Fling BW, Peltier SJ, Bo J, Welsh RC, Seidler RD: Age differences in interhemispheric interactions: callosal structure, physiological function, and behavior. Front Neurosci 2011; 5:38.

18 Langan J, Peltier SJ, Bo J, Fling BW, Welsh RC, Seidler RD: Functional implications of age differences in motor system connectivity. Front Syst Neurosci 2010;4:17.

19 Antonenko D, Meinzer M, Lindenberg R, Witte AV, Floel A: Grammar learning in older adults is linked to white matter microstructure and functional connectivity. Neuroimage 2012;62:1667-1674.

20 Antonenko D, Brauer J, Meinzer M, Fengler A, Kerti L, Friederici AD, Flöel A: Functional and structural syntax networks in aging. Neuroimage 2013;83C:513-523.

21 Salami A, Eriksson J, Nyberg L: Opposing effects of aging on large-scale brain systems for memory encoding and cognitive control. J Neurosci 2012;32:10749-10757.
22 Spreng RN, Schacter DL: Default network modulation and large-scale network interactivity in healthy young and old adults. Cereb Cortex 2012;22:2610-2621.

23 Davis SW, Kragel JE, Madden DJ, Cabeza R: The architecture of cross-hemispheric communication in the aging brain: linking behavior to functional and structural connectivity. Cereb Cortex 2012;22:232-242.

24 Chen NK, Chou YH, Song AW, Madden DJ: Measurement of spontaneous signal fluctuations in fMRI: adult age differences in intrinsic functional connectivity. Brain Struct Funct 2009;213:571-585.

-25 Lövdén M, Schmiedek F, Kennedy KM, Rodrigue KM, Lindenberger U, Raz N: Does variability in cognitive performance correlate with frontal brain volume? Neuroimage 2013; 64:209-215.

26 Madden DJ, Whiting WL, Huettel SA, White LE, MacFall JR, Provenzale JM: Diffusion tensor imaging of adult age differences in cerebral white matter: relation to response time. Neuroimage 2004;21:1174-1181.

27 Friederici AD: The brain basis of language processing: from structure to function. Physiol Rev 2011;91:1357-1392.

28 Turi Z, Paulus W, Antal A: Functional neuroimaging and transcranial electrical stimulation. Clin EEG Neurosci 2012;43:200-208.

29 Meinzer M, Antonenko D, Lindenberg R, Hetzer S, Ulm L, Avirame K, Flaisch T, Flöel A: Electrical brain stimulation improves cognitive performance by modulating functional connectivity and task-specific activation. J Neurosci 2012;32:1859-1866.

30 Meinzer M, Lindenberg R, Antonenko D, Flaisch T, Flöel A: Anodal transcranial direct current stimulation temporarily reverses ageassociated cognitive decline and functional brain activity changes. J Neurosci 2013;33: 12470-12478. 